\title{
A Packet-Switching Strategy for Uncertain Nonlinear Networked Control Systems *
}

\author{
Luca Greco*, Antoine Chaillet ${ }^{* *}$, Antonio Bicchi ${ }^{* * *}$ \\ *L2S - EECI - Supélec. (e-mail: lgreco@ieee.org) \\ ** L2S - EECI - Univ. Paris Sud 11 - Supélec. 3 rue Joliot-Curie. \\ 91192 - Gif sur Yvette, France (e-mail: chaillet@ieee.org). \\ *** Interdep. Research Center "E. Piaggio", University of Pisa, Italy; \\ IIT - Istituto Italiano di Tecnologia, Genova, Italy (e-mail: \\ bicchi@ing.unipi.it).
}

\begin{abstract}
This paper addresses the problem of stabilizing uncertain nonlinear plants over a shared limited-bandwidth packet-switching network for which both the time between consecutive accesses to each node (MATI) and the transmission and processing delays (MAD) for measurements and control packets are bounded. While conventional control loops are designed to work with circuit-switching networks, where dedicated communication channels provide almost constant bit rate and delay, many networks, such as Ethernet, organize data transmission in packets, carrying larger amount of information at less predictable rates. To avoid the bandwidth waste due to the relatively large overhead inherent to packet transmission, we exploit the packet payload to carry longer control sequences. To this aim we adopt a model-based approach to remotely compute a predictive control signal on a suitable time horizon, which leads to effectively reducing the bandwidth required to guarantee stability. Communications are assumed to be ruled by a rather general protocol model, which encompasses many protocols used in practice. As a distinct improvement over the state of the art, our result is shown to be robust with respect to sector-bounded uncertainties in the plant model. Namely, an explicit bound on the combined effects of MATI and MAD is provided as a function of the basin of attraction and the model accuracy.
\end{abstract}

Keywords: Networked control systems, packet-switching networks, model-based control, time-varying delays.

\section{INTRODUCTION}

A Networked Control System (NCS) is a system in which sensors, actuators and controllers are spatially distributed and exchange information through a shared, digital, finite capacity channel. The use of the network as a communication medium and the distributed nature of the system make traditional control theory not always applicable. Issues such as quantization errors, data dropouts, variable transmission intervals, variable communication delays, and constrained access to the network, can no longer be ignored (Hespanha et al. (2007)). The NCS literature has separately addressed many of these problems, and sometimes the combinations thereof. An excellent discussion of the state-of-the-art is reported in Heemels et al. (2010), and the reader is referred there for a detailed analysis of the literature towards the mentioned communication constraints. An essential aspect of NCS is the packet-switching nature of many networks. As opposed to conventional control loops, which are designed to work with circuitswitching networks where dedicated communication channels provide almost constant bit rate and delay, networks such as Ethernet organize data transmission in packets, carrying larger amount of information at less predictable rates.

\footnotetext{
‡ This work was partially supported by the HYCON2 NoE, contract number FP7-ICT-257462, and by the Contract IST 224428 (2008) (STREP) "CHAT - Control of Heterogeneous Automation Systems: Technologies for scalability, reconfigurability and security".
}

The organization of control information in data packets, which have relatively large transmission overhead, substantially alter the bandwidth/performance trade-off of traditional design. For instance, important data-rate theorems (see e.g. Hespanha et al. (2002), Nair and Evans (2003), Nair and Evans (2004)) expressing a fundamental relationship between the degree of instability of a given physical system and the minimum bit rate required to stabilize it, do not account for the fact that data come in packets with a minimum size (e.g. 84 bytes in Ethernet). To simplify, transmitting a 16 bits record every millisecond requires as much bandwidth in average as sending a packet of 84 bytes every 48 milliseconds; however, the implications on the effective sampling rate and feedback control performance are apparent. How to recover part of this performance is an objective of this study.

A second aspect inherent to packet-switching networks is transmission overhead. For instance, every Ethernet packet carries 38 bytes of headers and interframe separations, and useless information is necessarily padded into the payload to reach the minimum required packet length. As a consequence, transmitting a few bits per packet has essentially the same bandwidth cost as transmitting hundreds of them. A new, specific trade-off hence arises between packet rate and packet dimension for a given estimation/control task.

While the above aspects have been observed and described in the early literature on NCS (see e.g. the surveys by Walsh and Ye (2001), Lian et al. (2001), Hespanha et al. (2007)), only recently have appeared results which address 
them explicitly in controller design. The goal can be succinctly described as to decrease the network utilization (in terms of bandwidth, or packets per unit of time) without compromising control performance. To achieve this, Montestruque and Antsaklis (2004) pioneered the idea of exploiting the empty portion of packet payload to carry feedforward control sequences, computed in advance on the basis of a model-based scheme. Following developments along these lines generalized the technique to address nonlinear systems (Quevedo et al. (2007)), time-varying delays and packet dropouts (Polushin et al. (2008), Pin and Parisini (2009)), as well as the constraints imposed by communication protocols on state measurement access (Chaillet and Bicchi (2008)).

In this paper we also adopt the feedforward approach to send in a packet not only the control value to be applied at a specific instant, but also a prediction of the control law valid on a given time-horizon, so as to better exploit the payload. In the same spirit of the above model-based approaches, the control sequence is obtained by simulating an (imprecise) model of the closed-loop plant. The internal state of the model is asynchronously updated by means of the measurements of the plant state provided by sensors. Due to their spatial distribution, only portions of the model state can be updated in each instant. Therefore, we consider the constrained access to the network to be ruled by a protocol deciding which sensor node can communicate at each instant. The large control-packet, sent by the remote controller, is stored in an embedded memory on the plant side. Based on a local re-synchronization, made possible by a time-stamping of measurements, this strategy also allows to compensate the effect of bounded communication delays in the control loop. We build our model upon the powerful hybrid formalism introduced in Nešić and Teel (2004), and we consider network imperfections affecting both sides of the control loop. We provide explicit bounds on the Maximum Allowable Delay (MAD) (see Heemels et al. (2010)) and on the Maximum Allowable Transfer Interval (MATI) (see Walsh et al. (1999), i.e. the maximum duration between two successive communications) ensuring the semiglobal exponential stability of the NCS.

The main contribution of this paper is a control strategy for packet-switching networks ensuring the stability of an uncertain nonlinear NCS affected by varying transmission intervals, varying (and potentially large) delays, and constrained access to the network. Unlike the commonly assumed small-delays hypothesis (see for instance Heemels et al. (2010)), we can compensate for delays larger than the transmission interval.

A line of work close to ours is reported in Polushin et al. (2008), where the problem of stabilizing a nonlinear NCS with feedforward control sequences is addressed. Such sequences are computed by means of an approximate discrete-time plant model. Authors assume that the approximation algorithm is the only source of uncertainty in the model and that the inaccuracy of such a model can be reduced at will in order to achieve the desired MATI. In this paper, instead, we consider a robustness problem, where the plant uncertainty is a given, and we provide a bound on the MATI in terms of the model inaccuracy (measured through its local Lipschitz constant).

Preliminary results concerning our approach were presented in Chaillet and Bicchi (2008). The present paper extends that in at least three relevant aspects. We consider

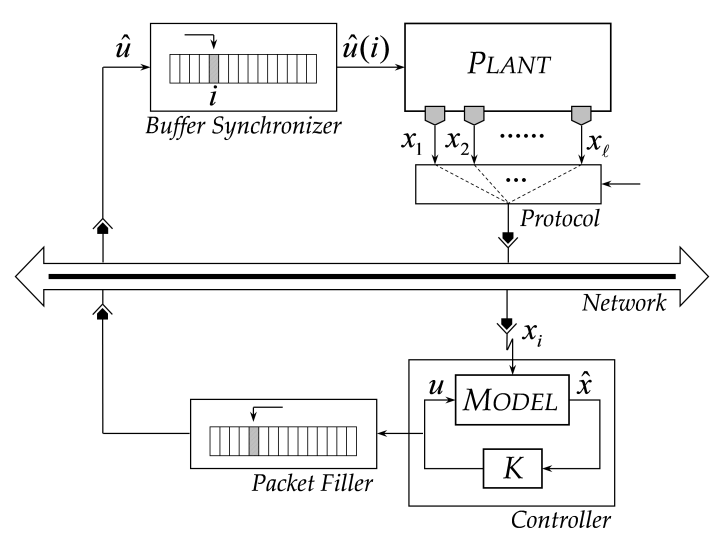

Fig. 1. Networked Control System with packet-switching network and protocol.

here uniformly global exponentially stable (UGES) protocols, rather than the conservative class of invariably UGES protocols (which do not include, for instance, the very common Round Robin protocol). We significantly extend the class of nonlinear plants by imposing only local Lipschitz conditions, instead of global ones. Finally, we take directly into account, in the computation of the MATI, the accuracy of the model used to build the prediction.

\section{PROBLEM STATEMENT}

\subsection{Network Model}

We consider a NCS constituted of a remote controller receiving measurements from and sending commands to a physical plant through a shared communication channel (see Figure 1). Control sequences are sent over the digital network as packets. An elementary embedded control device receives, decodes, synchronizes these packets and applies control commands to the plant. Measurements are taken by physically distributed sensors and sent towards the controller as packets encoded with sufficient precision to neglect quantization effects. Sensors are assumed to be embedded with the plant and hence synchronized with it. Due to the distributed nature of the sensors, we also assume that the measurement part of the network is partitioned in $\ell$ nodes and only a unique node at a time can send its information (i.e. only partial knowledge of the plant state is available at each time instants). We consider that measurements are taken and sent at instants $\left\{\tau_{i}^{m}\right\}_{i \in \mathbb{N}}$, and are received by the remote controller at instants $\left\{\tau_{i}^{m}+\right.$ $\left.T_{i}^{m}\right\}_{i \in \mathbb{N}}$. In other words, $\left\{T_{i}^{m}\right\}_{i \in \mathbb{N}}$ denote the (possibly time-varying) measurement data delays, which cover both processing and transmission delays on the measurement chain. In the same way, control commands are computed, encoded into packets and sent over the network at time instants $\left\{\tau_{j}^{c}\right\}_{j \in \mathbb{N}}$. They reach the plant at instants $\left\{\tau_{j}^{c}+\right.$ $\left.T_{j}^{c}\right\}_{j \in \mathbb{N}}$, where $\left\{T_{j}^{c}\right\}_{j \in \mathbb{N}}$ denote the (possibly time-varying) control data delays accounting both for computation and transmission delays from the controller to the plant.

Assumption 1. (Network) The communication network satisfies the following properties:

i) (MATI) There exist two constants $\tau_{m}, \tau_{c} \geq 0$ such that $\tau_{i+1}^{m}-\tau_{i}^{m} \leq \tau_{m}$ and $\tau_{j+1}^{c}-\tau_{j}^{c} \leq \tau_{c}, \forall i, j \in \mathbb{N}$;

ii) (MAD) There exist two constants $T_{m}, T_{c} \geq 0$ such that $T_{i}^{m} \leq T_{m}$ and $T_{j}^{c} \leq T_{c}, \forall i, j \in \mathbb{N}$

iii) (No Zeno phenomenon) There exist constants $\varepsilon_{m}>0$ and $\varepsilon_{c}>0$ such that $\varepsilon_{m} \leq \tau_{i+1}^{m}-\tau_{i}^{m}, \forall i \in \mathbb{N}$ and $\varepsilon_{c} \leq \tau_{j+1}^{c}-\tau_{j}^{c}, \forall j \in \mathbb{N}$. 
Item i) in the previous assumptions imposes that the MATI between two consecutive accesses to the network is bounded both for measurements and control. Item ii) imposes that the MADs, both on measurements and control side, are bounded. Item iii) imposes that the minimum time interval between two consecutive accesses to the network by the nodes is lower bounded away from zero, and similarly for the control side. The objective of this paper is to provide explicit bounds on the MATIs $\left(\tau_{m}\right.$ and $\left.\tau_{c}\right)$ and on the MADs $\left(T_{m}\right.$ and $\left.T_{c}\right)$ to guarantee exponential stability of the closed-loop NCS based on a specific control procedure.

\subsection{Protocol Model}

The access to the network is ruled by a protocol choosing, at each instant $\tau_{i}^{m}$, which node communicates its data. Decisions can be taken either according to the time index $i$ (static protocol) or based on the value of the error $e$ between the state estimate $\hat{x}$ and the available state measurements $x$ from sensors (dynamic protocol). More precisely, in the spirit of Nešić and Teel (2004), we model the network protocol as a time-varying discrete-time system involving the error $\mathbb{R}^{n} \ni e \triangleq \hat{x}-x, n \in \mathbb{N}_{\geq 1}$, that this type of communication generates:

$$
e(i+1)=h(i, e(i)), \quad \forall i \in \mathbb{N},
$$

where $h: \mathbb{N} \times \mathbb{R}^{n} \rightarrow \mathbb{R}^{n}$. If the network were able to send the measurement of the whole state at each time instant $\tau_{i}^{m}$, then the function $h$ would be identically zero; this is an assumption commonly posed in the literature on NCSs (see for instance Branicky et al. (2000), Zhivoglyadov and Middleton (2003), Montestruque and Antsaklis (2004), Seuret et al. (2005), Yue et al. (2005), Naghshtabrizi and Hespanha (2006), Quevedo et al. (2007), Polushin et al. (2008), Pin and Parisini (2009)) where network effects are mostly modeled as sampling and delays. This assumption may no longer be justified when sensors are physically distributed. It is worth noting that we assume the dynamics (1) to model only the communication error induced by the distributed nature of sensor nodes, while the modeling of delay effects is deferred to the next section. A different approach to the communication errors and protocol can be found in Heemels et al. (2010).

Purely static protocols involve a function $h$ which takes as an argument the time index $i$ only. An example of such protocols is the Round Robin (RR) protocol, which executes a cyclic inspection of each node. On the opposite, some network protocols purely rely on the current value of the error, in which case $h$ is independent of $i$ : this is the case of the Try-Once-Discard (TOD) protocol (Walsh et al. (1999)). The objective of most communication protocols is to decrease some function of the transmission error $e$ at each transmitted packet. A particularly relevant class of such protocols is the one that ensures an exponential decay of this error. We recall here a slightly modified version of the definition in Nešic and Teel (2004) to focus on the class of protocols we deal with in this work.

Assumption 2. (UGES Protocol) The protocol modeled by the discrete-time system (1) is uniformly globally exponentially stable (UGES) and admits an associated Lyapunov function with bounded gradient. That is, there exist a function $W_{0}: \mathbb{N} \times \mathbb{R}^{n} \rightarrow \mathbb{R}_{\geq 0}$ locally Lipschitz in the second argument, and constants $\underline{a}, \bar{a}, c>0$ and $\rho_{0} \in[0,1)$ such that, for all $e \in \mathbb{R}^{n}$ and all $i \in \mathbb{N}$,

$$
\underline{a}|e| \leq W_{0}(i, e) \leq \bar{a}|e|
$$

$$
W_{0}(i+1, h(i, e)) \leq \rho_{0} W_{0}(i, e),
$$

and for almost all $e \in \mathbb{R}^{n}$ and all $i \in \mathbb{N}$

$$
\left|\frac{\partial W_{0}}{\partial e}(i, e)\right| \leq c \text {. }
$$

It is worth stressing that the UGES protocols considered here are not necessarily invariably UGES, as assumed in Chaillet and Bicchi (2008). The latter property is rather restrictive, as it excludes, for instance, the commonly adopted Round Robin protocol.

\subsection{The plant and its model}

We assume that a nominal feedback controller is given, which would be able, in the absence of the effects induced by the network, to globally exponentially stabilize the real plant. More precisely, we assume the following.

Assumption 3. (Nominal GES) There exists a continuously differentiable function $\kappa: \mathbb{R}^{n} \rightarrow \mathbb{R}^{m}$ such that the closed-loop system

$$
\begin{aligned}
& \dot{x}=f(x, u) \\
& u=\kappa(x)
\end{aligned}
$$

is globally exponentially stable (GES), so that there exists a differentiable function $V: \mathbb{R}^{n} \rightarrow \mathbb{R}_{\geq 0}$ and constants $\underline{\alpha}, \bar{\alpha}, \alpha, d>0$ such that the following conditions hold for all $x \in \mathbb{R}^{n}$

$$
\begin{gathered}
\frac{\alpha}{V}|x|^{2} \leq V(x) \leq \bar{\alpha}|x|^{2} \\
\frac{\partial V}{\partial x}(x) f(x, \kappa(x)) \leq-\alpha|x|^{2} \\
\left|\frac{\partial V}{\partial x}(x)\right| \leq d|x|
\end{gathered}
$$

In order to compute the control signal, the remote controller makes use of a state estimate based on an approximate model $\hat{f}$ of the plant $f$. Both the plant and its model are considered to be zero at the origin $(f(0, \kappa(0))=$ $\hat{f}(0, \kappa(0))=0)$. The strategy developed in this paper relies on the assumption that the plant, its model and the nominal controller are all locally Lipschitz.

Assumption 4. (Local Lipschitz) Given some constants $R_{x}, R_{u}>0$, there exist some positive constants $\lambda_{f}$ and $\lambda_{\kappa}{ }^{1}$ such that for all $x_{1}, x_{2} \in B_{R_{x}}$ and all $u_{1}, u_{2} \in B_{R_{u}}$, the following inequalities hold

$$
\begin{aligned}
\left|f\left(x_{1}, u_{1}\right)-f\left(x_{2}, u_{2}\right)\right| & \leq \lambda_{f}\left(\left|x_{1}-x_{2}\right|+\left|u_{1}-u_{2}\right|\right) \\
\left|\kappa\left(x_{1}\right)-\kappa\left(x_{2}\right)\right| & \leq \lambda_{\kappa}\left|x_{1}-x_{2}\right| .
\end{aligned}
$$

It is worth noting that the previous assumption represents a further important relaxation with respect to Chaillet and Bicchi (2008), where all involved vector fields were assumed to be globally Lipschitz.

Finally, we assume that the plant model inaccuracy is sector-bounded.

Assumption 5. (Sector-Bounded Model Inaccuracy) Given $R_{x}, R_{u}>0$, there exists a nonnegative constant $\lambda_{f \hat{f}}$ such that for all $x \in B_{R_{x}}$ and all $u \in B_{R_{u}}$,

$$
|\hat{f}(x, u)-f(x, u)| \leq \lambda_{f \hat{f}}(|x|+|u|)
$$

The constant $\lambda_{f \hat{f}}$ thus measures the model accuracy: the closer the model $\hat{f}$ is to the real system $f$, the smaller is $\lambda_{f \hat{f}}$ (in the ideal case of perfect modeling, it would be zero). Note that Assumption 5 allows to cope with both parametric uncertainties and unmodeled dynamics.

\footnotetext{
1 We stress that $\lambda_{\kappa}$ can be chosen independently of $R_{u}$.
} 


\section{A MODEL-BASED STRATEGY}

\subsection{Modeling the overall setup}

We develop here a model-based strategy exploiting the relatively large payload of a packet. At each reception of a new measurement, the remote controller updates an estimate of the current state of the plant and computes a prediction of the control signal over a fixed time horizon $T_{0}^{p}$ by numerically running the model $\hat{f}$. This signal is then coded and sent in a single packet at the next network access. When received by the plant, it is decoded and resynchronized by the embedded computer, based on the time-stamping of the original measurement. We assume here that the plant and its sensors have a common clock; however, we also stress that in our strategy there is no need for clock synchronization between the plant and the remote controller.

In order to guarantee that a relevant control signal is always available, the fixed time horizon $T_{0}^{p}$ on which each state prediction is achieved is chosen as

$$
T_{0}^{p} \geq T_{c}+T_{m}+\tau_{m}+\tau_{c}
$$

This prediction horizon guarantees, in view of Assumption 1 , that a control sequence corresponding to the present time is always loaded in the embedded buffer.

For sake of mathematical rigor, we introduce first a model accounting for infinitely many state variables and infinitely many duplicates of the model $\hat{f}$. In Section 3.2, we show how to properly reduce them to a finite number. Therefore, for any measurement taken at $\tau_{i}^{m}, i \in \mathbb{N}$, we consider a new estimate state variable $\hat{x}_{i}$, valid over the time interval $\left[\tau_{i}^{m}, \tau_{i}^{m}+T_{0}^{p}\right]$, whose evolution is given by

$$
\begin{aligned}
\dot{\hat{x}}_{i}(t) & =\hat{f}\left(\hat{x}_{i}(t), \kappa\left(\hat{x}_{i}(t)\right)\right), \quad \forall t \in\left[\tau_{i}^{m}, \tau_{i}^{m}+T_{0}^{p}\right] \\
\hat{x}_{i}\left(\tau_{i}^{m+}\right) & =x\left(\tau_{i}^{m}\right)+h\left(i, \hat{x}_{i-1}\left(\tau_{i}^{m}\right)-x\left(\tau_{i}^{m}\right)\right) .
\end{aligned}
$$

Each variable is updated at time $\tau_{i}^{m+}$ according to the protocol $h$. Usually, when dealing with a unique variable, the update of an estimate is performed by means of the error between the measurement and the variable itself. In our case, instead, a new estimate variable $\hat{x}_{i}$ is created at each $\tau_{i}^{m}$, with the previous variable $\hat{x}_{i-1}$ containing the latest value of the estimate. Hence, the error we compute at time $\tau_{i}^{m}$ is between the measurement made on $x\left(\tau_{i}^{m}\right)$ and the previous estimate variable $\hat{x}_{i-1}\left(\tau_{i}^{m}\right)$. In this way all measurements are used to continuously update the internal model.

The infinite sequence of evolutions for the simulated dynamics (11) is schematically depicted at the top of Figure 2, above the time line. Each simulated evolution is represented by a straight line starting at times $\tau_{i}^{m}, i \in \mathbb{N}$ (explicitly reported at their left). Different line styles represent different evolutions for the estimate variables. The time line reports the instants $\tau_{i}^{m}+T_{i}^{m}, i \in \mathbb{N}$ at which the measurements $x\left(\tau_{i}^{m}\right)$ reach the controller. It is important to remark that the dynamics (11) actually evolves in a virtual (simulated) time. The measurement $x\left(\tau_{i}^{m}\right)$ reaches the controller at $\tau_{i}^{m}+T_{i}^{m}$ and then triggers the simulation of the dynamics (11) for a virtual time interval $\left[\tau_{i}^{m}, \tau_{i}^{m}+\right.$ $\left.T_{0}^{p}\right]$. The actual time spent for this simulation and for the computation of the predicted control signal is, in fact, part of the delay $T_{j}^{c}$. What we have done in (11), is to consider the estimate dynamics 'stretched' on the real time as if it ran concurrently with the plant. This notation trick allows us to cast the overall system in a compact model similar to the one in Nešić and Teel (2004).

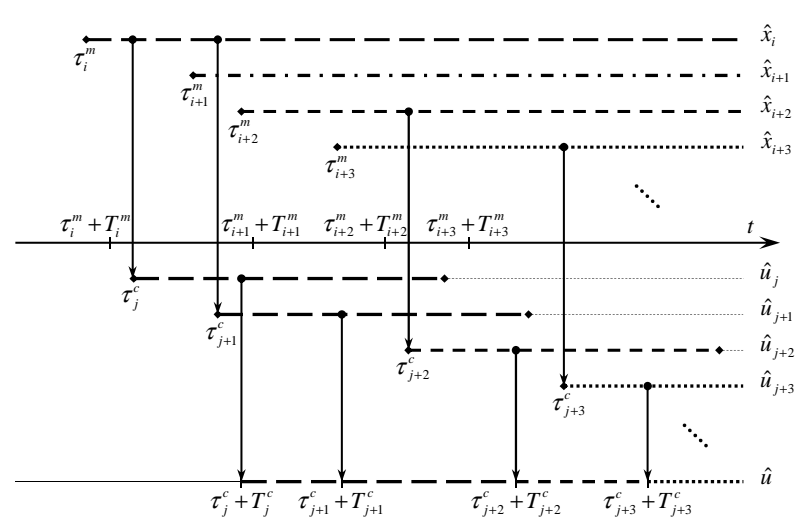

Fig. 2. Excerpt of a sequence of estimate variables and feedforward signals along with the control signal applied to the plant.

At each instant $\tau_{j}^{c}$ a new control signal $u_{j}(\cdot)$ is computed. It is based on the estimate variable $\hat{x}_{\gamma(j)}$, where $\gamma(j)$ denotes the index of the latest measurement received before $\tau_{j}^{c}$. More precisely, the function $\gamma: \mathbb{N} \rightarrow \mathbb{N}$ is defined as

$$
\gamma(j) \triangleq \max \left\{i \in \mathbb{N} \mid \tau_{i}^{m}+T_{i}^{m}<\tau_{j}^{c}\right\}, \quad \forall j \in \mathbb{N} .
$$

It can be easily verified that, in view of Assumption 1, the time horizon $T_{0}^{c}$ for the control signal has to satisfy

$$
T_{0}^{c} \geq T_{c}+\tau_{c}
$$

in order to guarantee that a valid control signal is always available to the embedded controller. Note that the required time horizon $T_{0}^{c}$ for the control signal is smaller than the time horizon $T_{0}^{p}$ used for prediction, as it does not need to account for measurement MAD and MATI. We thus define an infinite number of feedforward control signals as

$$
\hat{u}_{j}(t)=\kappa\left(\hat{x}_{\gamma(j)}(t)\right), \quad \forall t \in\left[\tau_{j}^{c}, \tau_{j}^{c}+T_{0}^{c}\right], \forall j \in \mathbb{N} .
$$

At each reception of a new control packet (i.e. at instants $\left.\tau_{j}^{c}+T_{j}^{c}\right)$, the embedded buffer is updated. Consequently, the control signal applied to the plant is given by

$$
\hat{u}(t)=\hat{u}_{j}(t), \quad \forall t \in\left[\tau_{j}^{c}+T_{j}^{c}, \tau_{j+1}^{c}+T_{j+1}^{c}\right) .
$$

Both the feedforward signals $\hat{u}_{j}$ and the control $\hat{u}$ are depicted at the bottom of Figure 2. Line styles are consistent with those of the estimate evolutions used to build the control signals. Vertical arrows show which estimate variable $\hat{x}_{\gamma(j)}$ is chosen for the computation of the feedforward signal $\hat{u}_{j}$ at time instant $\tau_{j}^{c}$, and which control signal $\hat{u}_{j}$ is used at $\tau_{j}^{c}+T_{j}^{c}$ to update the embedded controller. In the particular example of Figure 2, it can be noticed that $\hat{u}_{j}$ and $\hat{u}_{j+1}$ are computed with respect to the same estimate $\hat{x}_{i}$ since $\gamma(j)=\gamma(j+1)=i$. On the other hand, $\hat{x}_{i+1}$ is not directly used by any control since $\gamma(j+2)=i+2$.

\subsection{A reduced NCS model}

The model considered so far makes use of infinitely many state estimate variables $\hat{x}_{i}$ and control signals $\hat{u}_{j}$. They can be reduced to a finite number by noticing that they are all defined over compact time intervals and that "old" variables are no longer used after a while. State estimates variables are stored in a finite memory and new values are cyclically written on dismissed variables. We must prevent that a variable is accidentally reset while still in use for the computation of a control signal. In particular, $\hat{x}_{\gamma(j)}$ cannot be reset during the interval $\left[\tau_{\gamma(j)}^{m}, \tau_{j+1}^{c}+T_{j+1}^{c}\right]$. 
Hence, the dimension of such a memory, in terms of number of variables, is given by the maximum number of measurements that can be received during the life horizon $T_{0}^{p}$ of an estimate variable. Recalling that $T_{0}^{p}$ accounts also for the interval during which no measurements are received, whose length is bounded by $\tau_{m}$, the dimension $N$ of the memory is given by

$$
N \triangleq\left\lfloor\frac{T_{0}^{p}-\tau_{m}}{\varepsilon_{m}}\right\rfloor+1 .
$$

Therefore, we use only $N$ state variables $x_{c_{r}}, r \in$ $\{1, \ldots, N\}$, to store the state estimates. They are cyclically updated according to the following relation

$$
x_{c_{r}}(t) \triangleq \hat{x}_{i}(t) \quad \text { iff } \eta(t, i, r)=1 \text {, }
$$

where $\eta: \mathbb{R}_{\geq 0} \times \mathbb{N} \times\{1, \ldots, N\} \rightarrow\{0,1\}$ is the function defined as

$$
\eta(t, i, r) \triangleq\left\{\begin{array}{l}
1 \text { if } t \in\left(\tau_{i}^{m}, \tau_{i+1}^{m}\right] \text { and } \mu(i)=r \\
0 \text { otherwise, }
\end{array}\right.
$$

which identifies the index of the relevant state estimate, and $\mu: \mathbb{N} \rightarrow\{1, \ldots, N\}$ is defined as

$$
\mu(i) \triangleq((i-1) \bmod N)+1
$$

to make a cyclic update of the state estimates in the memory. By means of the vectors $\bar{x}, x_{c}, e \in \mathbb{R}^{N n}$ defined as $\bar{x} \triangleq\left[x^{T}, \ldots, x^{T}\right]^{T}, x_{c} \triangleq\left[x_{c_{1}}^{T}, \ldots, x_{c_{N}}^{T}\right]^{T}$ and $e=$ $\left[e_{1}^{T}, \ldots, e_{N}^{T}\right]^{T} \triangleq x_{c}-\bar{x}$, the closed-loop dynamics of the NCS can be compactly written as

$$
\begin{aligned}
\dot{x} & =F(t, \bar{x}, e) \\
\dot{e} & =G(t, \bar{x}, e) \\
e\left(\tau_{i}^{m+}\right) & =H\left(i, e\left(\tau_{i}^{m}\right)\right),
\end{aligned}
$$

where

$$
\begin{aligned}
F(t, \bar{x}, e) & =f(x, u(t, e+\bar{x})) \\
G(t, \bar{x}, e) & =\left[\begin{array}{c}
\hat{f}\left(e_{1}+x, \kappa\left(e_{1}+x\right)\right)-f(x, u(t, e+\bar{x})) \\
\vdots \\
\hat{f}\left(e_{N}+x, \kappa\left(e_{N}+x\right)\right)-f(x, u(t, e+\bar{x}))
\end{array}\right]
\end{aligned}
$$$$
H(i, e)=\left[\begin{array}{c}
e_{1}+\left(h\left(i, e_{N}\right)-e_{1}\right) \eta(t, i, 1) \\
e_{2}+\left(h\left(i, e_{1}\right)-e_{2}\right) \eta(t, i, 2) \\
\vdots \\
e_{N}+\left(h\left(i, e_{N-1}\right)-e_{N}\right) \eta(t, i, N)
\end{array}\right]
$$

The control signal $u$ in (18a) and (18b) is given by ${ }^{2}$

$$
u\left(t, x_{c}\right) \triangleq \sum_{k=1}^{N} \kappa\left(x_{c_{k}}\right) \nu(t, j, k), \quad \forall j \in \mathbb{N},
$$

where the function $\nu: \mathbb{R}_{\geq 0} \times \mathbb{N} \times\{1, \ldots, N\} \rightarrow\{0,1\}$ is defined as

$$
\nu(t, j, k) \triangleq \begin{cases}1 & \text { if } t \in\left(\tau_{j}^{c}+T_{j}^{c}, \tau_{j+1}^{c}+T_{j+1}^{c}\right] \\ 0 & \text { and } \mu(\gamma(j))=k\end{cases}
$$

This compact notation carries the advantage to involve a finite number of state variables and to fit the framework of Nešic and Teel (2004). Note that the control signal in (13) now reads $\hat{u}(t)=u\left(t, x_{c}(t)\right)$.

\section{MAIN RESULTS}

We start by stating that the obtained protocol (17c) and (18c) inherits the UGES property from the original one

2 Since $\nu(t, j, k) \neq 0$ only when $\mu(\gamma(j))=k$, the control input in (19) is independent of $j$ contrarily to what the notation suggests.
(1). Due to space constraints proofs have been omitted and can be found in Greco et al. (2010).

Proposition 1. Under Assumption 2, the protocol modeled by the discrete-time system (17c) and (18c) is UGES and admits an associated Lyapunov function $W: \mathbb{N} \times$ $\mathbb{R}^{N n} \rightarrow \mathbb{R}_{\geq 0}$ given by

$$
W(k, e) \triangleq \sum_{r=1}^{N} W_{0}\left(k, e_{r}\right) \eta(t, k, r)
$$

where $\eta$ is defined in (15), and satisfying for all $k \in \mathbb{N}$ and all $e \in \mathbb{R}^{N n}$ :

$$
\begin{gathered}
a_{L}|e| \leq W(k, e) \leq a_{H}|e| \\
W(k+1, H(k, e)) \leq \rho_{0} W(k, e) \\
\left|\frac{\partial W}{\partial e}(k, e)\right| \leq c,
\end{gathered}
$$

with $a_{L} \triangleq \underline{a}$ for $N=1$ and $a_{L} \triangleq \frac{a}{N} \min \left\{1,\left(\frac{\underline{a}}{\bar{a}}\right)^{2} \frac{1}{\rho_{0}}\right\}$ for $N>1$, and $a_{H} \triangleq \bar{a}$.

Let us now present a local result on the exponential stability of the NCS (17). It provides an explicit bound (cf. (23) below) on the measurement MATI $\tau_{m}$ in terms of the characteristic parameters of the network-free closedloop system, the protocol, the regularity assumptions on the dynamics and the model precision.

Theorem 1. Assume that Assumptions 1-3 hold. Given some $R>0$, fix $R_{x}=R$ and $R_{u}=\lambda_{\kappa} R$ and suppose that Assumptions 4-5 hold with these constants. Let $\underline{a}, \bar{a}$, $\rho_{0}, c, \underline{\alpha}, \bar{\alpha}, \alpha, d, \lambda_{f \hat{f}}, \lambda_{f}, \lambda_{\kappa}, a_{L}, a_{H}$ be generated by these assumptions and by Proposition 1. Assume that the following conditions on $\tau_{m}, \tau_{c}, T_{m}, T_{c}, \varepsilon_{m}$ hold

$$
\begin{aligned}
& \tau_{m} \in\left[\varepsilon_{m}, \tau_{m}^{\star}\right), \tau_{m}^{\star} \triangleq \frac{1}{L} \ln \left(\frac{H \gamma_{2}+a_{L} L}{H \gamma_{2}+a_{L} \rho_{0} L}\right) \\
& N=\left\lfloor\frac{T_{c}+T_{m}+\tau_{c}}{\varepsilon_{m}}\right\rfloor+1
\end{aligned}
$$

where $\quad L \quad \triangleq \quad \frac{c}{a_{L}}\left(\sqrt{N}\left(\lambda_{f \hat{f}}\left(1+\lambda_{\kappa}\right)+\lambda_{f}\right)+\right.$ $\left.(\sqrt{N-1}+N-1) \lambda_{f} \lambda_{\kappa}\right), H \triangleq c N \lambda_{f \hat{f}}\left(1+\lambda_{\kappa}\right)$ and $\gamma_{2} \triangleq$ $\frac{d}{\alpha} \sqrt{\frac{\bar{\alpha}}{\underline{\alpha}}} \lambda_{f} \lambda_{\kappa}$. Then, the origin of the NCS (17) is exponentially stable with radius of attraction

$$
\tilde{R}=\frac{R}{K}
$$

where $K \triangleq \frac{\sqrt{2}}{1-\gamma_{1} \gamma_{2}} \max \left\{k_{2}\left(1+\gamma_{1}\right), k_{1}\left(1+\gamma_{2}\right)\right\}, \gamma_{1} \triangleq$ $\frac{\exp \left(L \tau_{m}\right)-1}{a_{L} L\left(1-\rho_{0} \exp \left(L \tau_{m}\right)\right)} H, k_{1} \triangleq \frac{a_{H}}{\rho_{0} a_{L}}$ and $k_{2} \triangleq \sqrt{\frac{\bar{\alpha}}{\underline{\alpha}}}$.

It is important to remark that the bound (23) on the measurement MATI is also related to the dimension of the memory $N$, whose definition (24), obtained by (14) for $T_{0}^{p}=T_{c}+T_{m}+\tau_{m}+\tau_{c}$, embeds the other relevant communication parameters: MADs and control MATI. The pair (23)-(24) thus imposes a trade-off between the two MATIs and the MADs. The packet-based strategy aims at enlarging the control MATI $\tau_{c}$, but a larger $\tau_{c}$ could require a larger memory $N$ and hence could produce a lower measurement MATI $\tau_{m}$. Moreover, conditions (23)(24) bind the four relevant parameters (i.e. $T_{c}, T_{m}, \tau_{c}$ and $\left.\tau_{m}\right)$ together and with the constant $\varepsilon_{m}$, bounding the minimum time between two consecutive accesses to the network. Furthermore, depending on the parameter $R$ for which Assumptions 4 and 5 hold, an explicit estimate $\tilde{R}$ 
of the radius of attraction can be computed, cf. (25). Note that, since Theorem 1 guarantees only local properties, Assumption 3 could be relaxed to local exponential stability of the nominal plant, over a sufficiently large domain. It can be shown (see Greco et al. (2010)) that the MATI and memory requirements of the previous theorem can always be satisfied.

In general, the radius of attraction $\tilde{R}$ of the resulting NCS guaranteed by Theorem 1 cannot be arbitrarily specified due to the possible dependency of the constants $L$ and $H$ (and consequently $K$ ) in the parameter $R$ ruling the domain on which Assumptions 4 and 5 hold. To see this more clearly, consider, for instance, the case of $K$ proportional to $R$. Relation (25) shows that, in this case, the radius of the initial condition $\tilde{R}$ would be a constant irrespective of the amplitude of $R$. One could even imagine that, in some situations, $\tilde{R}$ actually shrinks when $R$ is enlarged. Hence, in order to ensure that the set of initial conditions can be arbitrarily enlarged, we must add some constraints on the growth rate of the constant $K$ or, equivalently, on some of the Lipschitz constants. After reporting a definition of semiglobal exponential stability which is adapted to our NCS framework, we present our main result in this regard in Theorem 2 .

Definition 1. The NCS (17) is said to be semiglobally exponentially stable if, for any $\tilde{R}>0$, there exist positive constants $\tau_{m}^{\star}(\tilde{R}), \tau_{c}^{\star}(\tilde{R}), T_{m}^{\star}(\tilde{R}), T_{c}^{\star}(\tilde{R})$ and $\varepsilon_{m}^{\star}(\tilde{R})$, as introduced in Assumption 1, such that its origin is exponentially stable on $B_{\tilde{R}}$.

Theorem 2. Suppose that Assumptions 1-4 hold for all $R_{x}, R_{u}>0$ and that there exists $\sigma \in(0,1)$ such that

$$
\lim _{s \rightarrow \infty} \frac{\lambda_{f}(s) \lambda_{\kappa}(s)}{s^{\sigma}}<\infty .
$$

Then, the NCS (17) is semiglobally exponentially stable.

The above result guarantees that, provided sufficient regularity of the dynamics involved (i.e. Lipschitz constants sublinear in the size of the domain over which they are computed), any prescribed compact domain of attraction can be reached if MADs and MATIs are small enough.

\section{CONCLUSIONS}

The problem of stabilizing nonlinear time-invariant plants over a limited-bandwidth packet-switching network has been considered. We presented a model-based approach to remotely compute a predictive control signal on a given time horizon. We considered a robustness problem, where the plant uncertainty is given a priori, and we provided a bound on the combined effects of the MATI and MAD as a function of the basin of attraction and the model precision. Our future research will focus on the exploitation of the packetization of measurements to further reduce the bandwidth occupation and to better cope with model parameter variations.

\section{REFERENCES}

Branicky, M.S., Phillips, S.M., and Zhang, W. (2000). Stability of networked control systems: Explicit analysis of delay. In Proc. American Control Conf., 2352-2357. Chicago, USA.

Chaillet, A. and Bicchi, A. (2008). Delay compensation in packet-switching networked controlled systems. In Proc. IEEE Int. Conf. on Decision and Control, 3620-3625.
Greco, L., Chaillet, A., and Bicchi, A. (2010). Exploiting packet size in uncertain nonlinear networked control systems. Preprint submitted to Automatica. URL http://hal-supelec.archives-ouvertes.fr/ hal-00583525/fr/.

Heemels, W.P.M.H., Teel, A.R., van de Wouw, N., and Nešić, D. (2010). Networked control systems with communication constraints: Tradeoffs between transmission intervals, delays and performance. IEEE Trans. on Automat. Contr., 55(8), 1781-1796.

Hespanha, J.P., Naghshtabrizi, P., and Xu, Y. (2007). A survey of recent results in networked control systems. Proc. of IEEE, 95(1), 138-162.

Hespanha, J.P., Ortega, A., and Vasudevan, L. (2002). Towards the control of linear systems with minimum bit-rate. In Proc. of the Int. Symp. on the Mathematical Theory of Networks and Syst.

Lian, F.L., Moyne, J.R., and Tilbury, D.M. (2001). Performance evaluation of control networks: Ethernet, ControlNet, and DeviceNet. IEEE Contr. Syst. Mag., 21(1), 66-83.

Montestruque, L.A. and Antsaklis, P. (2004). Stability of model-based networked control systems with timevarying transmission times. IEEE Trans. on Automat. Contr., 49(9), 1562-1572.

Naghshtabrizi, P. and Hespanha, J.P. (2006). Stability of networked control systems with variable sampling and delay. In Proc. of the Annual Allerton Conf. on Communication, Control, and Computing.

Nair, G.N. and Evans, R.J. (2003). Exponential stabilisability of finite-dimensional linear systems with limited data rates. Automatica, 39(4), 585-593.

Nair, G.N. and Evans, R.J. (2004). Stabilizability of stochastic linear systems with finite feedback data rates. SIAM J. Contr. Optim., 43(2), 413-436.

Nešić, D. and Teel, A.R. (2004). Input-output stability properties of networked control systems. IEEE Trans. on Automat. Contr., 49(10), 1650-1667.

Pin, G. and Parisini, T. (2009). Networked predictive control of constrained nonlinear systems: Recursive feasibility and input-to-state stability analysis. In Proc. American Control Conf., 2327-2334. St. Louis, MO, USA.

Polushin, I., Liu, P., and Lung, C.H. (2008). On the modelbased approach to nonlinear networked control systems. Automatica, 44(9), 2409-2414.

Quevedo, D.E., Silva, E.I., and Goodwin, G.C. (2007). Packetized predictive control over erasure channels. In Proc. American Control Conf., 1003-1008. NY City, USA.

Seuret, A., Fridman, E., and Richard, J.P. (2005). Exponential stabilization of delay neutral systems under sampled-data control. In Proc. 13th Mediterranean Conference on Control and Automation. Limassol, Cyprus.

Walsh, G.C. and Ye, H. (2001). Scheduling of networked control systems. IEEE Contr. Syst. Mag., 21(1), 57-65.

Walsh, G.C., Ye, H., and Bushnell, L. (1999). Stability analysis of networked control systems. In Proc. American Control Conf.

Yue, D., Han, Q.L., and Lam, J. (2005). Networkedbased robust $H_{\infty}$ control of systems with uncertainty. Automatica, 41, 999-1007.

Zhivoglyadov, P.V. and Middleton, R.H. (2003). Networked control design for linear systems. Automatica, $39,743-750$. 